UOSTP-03-105

SNUTP 03-022

hep-th/0310123

\title{
Troubles with Spacetime Noncommutative Theories: Tachyons or S-branes?
}

\author{
Dongsu Bak \\ Physics Department, University of Seoul, Seoul 130-743, Korea \\ dsbak@mach.uos.ac.kr
}

\section{Seok Kim \\ School of Physics, Seoul National University, Seoul 151-747, Korea calaf2@snu.ac.kr}

\begin{abstract}
We find Lorentzian solutions of spacetime noncommutative gauge theories that are localized exponentially in space and time. Together with time translational invariance of the theories, we argue that perurbative $\mathrm{S}$ matrix formulation of such theories is problematic in the sense that the $\mathrm{S}$ matrix based on free in and out states misses the spacetime localized degrees. We show that, in $3+1$ dimensions, the problem disappears for the cases where the noncommutativity becomes purely spatial by an appropriate Lorentz transformation or the noncommutativity is lightlike with its electric and magnetic parts orthogonal to each other.
\end{abstract}




\section{Contents}

1. Introduction 1

2. Spacetime Localized Lorentzian Solutions 2

3. Discussions 6

\section{Introduction}

There have been some discussions about whether spacetime noncommutative theories are well defined as quantum theories [1]- [9]. At first, it was argued that spacetime noncommutative theories have a unitarity problem by studying the S-matrices via the perturbative analysis [1, 2, 3]. This is consistent with the fact that there is no decoupling limit from string theories leading to the spacetime noncommutative field theories for specific ranges of the noncommutativity parameter [1, 2n. Later it was claimed that one may recover the perturbative unitarity by the careful treatment of time orderings $\left[\begin{array}{l}\text {, } \\ \text { [ }\end{array}\right.$. There have been further studies [6, [7] in this direction but the status of the matter seems not clear at this moment.

The spacetime noncommutative field theories involve infinitely many time derivatives. There is no well established procedure by which one can define quantization of such theories. In the perturbative analysis, Feynman rules are given as a definition of the quantum theory but its justification is never clear.

In this note, we shall consider spacetime noncommutative field theories only classically. By solving equations of motion with Lorentzian signature, we shall construct solutions that are exponentially localized in time as well as spatial directions. Due to the translational invariance of the theory, one may find such solutions centered at any points in spacetime once there exist any. The existence of such solution implies that arbitrarily small fluctuations in the far past may become nontrivial ones of order one in the far future when such objects are translated to the far future. To put an emphasis on this, we shall show that such spacetime localized degrees may arise not just from the free vacuum but also from scattering states by explicitly constructing solutions of spacetime localized degrees superposed with scattering states. Perturbative S matrix formulation of the spacetime noncommutative theory will be problematic because the S-matrix based on free part degrees of freedom would miss the spacetime localized degrees. More precisely, scattering states alone and scattering states with spacetime localized degrees are physically distinct but cannot be distinguished, in the far past, as an initial data. Nonperturbative treatment of the system may cure the problem but we do not know how to do this. 
We shall illustrate existence of spacetime localized solutions by considering various noncommutative gauge theories in $1+1$ and $3+1$ dimensions for certain choice of noncommutative parameters $\theta^{\mu \nu}$ with nonvanishing electric components. When the noncommutativity is purely spatial, it is quite clear that there is no problem of unitarity at least classically and the standard procedure of quantization works without any troubles. As in [1, 2], Lorentz invariant combinations of $\theta^{\mu \nu}$ are relevant here. Specifically for $3+1$ dimensions, the cases of $\vec{\theta}_{e} \cdot \vec{\theta}_{m}=0$ and $\vec{\theta}_{e}^{2} \leq \vec{\theta}_{m}^{2}$ with $\theta_{e}^{i}=\theta^{0 i}$ and $\theta_{m}^{i}=\frac{1}{2} \epsilon^{i j k} \theta^{j k}$ will be free of problems. Otherwise the gauge theories are plagued with the Lorentzian solutions that are localized both in space and time. For the $\theta^{\mu \nu}$ without such problematic solutions, string theories in the NS-NS B-field background allow the decoupling field-theory limit[1], 2]. Therefore we get an agreement.

All of these solutions are closely related to the solitons, vortices, and instantons in the noncommutative gauge theories with spatial noncommutativity 10, 11, 12, 13, 14, 15, 16]. With purely spatial noncommutativity, the string theory interpretation of the solution is known. For example, the vortex solution in $2+1$ dimensional U(1) gauge theory corresponds to unstable D0 in D2 with NS-NS B fields 12. In the present case, we do not find such an interpretation. If they were Euclidean solutions, one might interpret them as instantons, but here we are dealing with Lorentzian solutions.

In Section 2, we shall construct the spacetime localized solutions in $1+1$ and $3+1$ dimensional gauge theories interacting with scalars on various noncommutative spacetimes. We won't try to find all of such solutions because finding only one will be enough to say that the theory is problematic. For $\mathrm{U}(1)$ or $\mathrm{U}(\mathrm{N})$ gauge theories 17], one may construct such solutions once the conditions on $\theta^{\mu \nu}$ are met. Section 3 will be devoted to discussions. We give an interpretation of the solutions and comment on the spacetime noncommutative scalar theories.

\section{Spacetime Localized Lorentzian Solutions}

We will present various theories and solutions in terms of ordinary functions equipped with the star product

$$
\left.f(x) * g(x) \equiv e^{\frac{i}{2} \theta^{\mu \nu} \partial_{\mu} \partial_{\nu}^{\prime}} f(x) g\left(x^{\prime}\right)\right|_{x=x^{\prime}} .
$$

There is a one-to-one correspondence between ordinary function and operator, where operators act on the Hilbert space built upon the commutation relations,

$$
\left[\hat{x}^{\mu}, \hat{x}^{\nu}\right]=i \theta^{\mu \nu}
$$

With the map realizing the correspondence,

$$
\hat{f}(\hat{x})=\int \frac{d^{D} k d^{D} x}{(2 \pi)^{D}} f(x) e^{i k \cdot(\hat{x}-x)},
$$

the operator product can be translated to the star product in the function space.

Let us consider the $1+1$ dimensional spacetime noncommutative $\mathrm{U}(1)$ gauge theory,

$$
S=-\frac{1}{4 g^{2}} \int d^{2} x F_{\mu \nu} * F^{\mu \nu}
$$


where

$$
F_{\mu \nu}=\partial_{\mu} A_{\nu}-\partial_{\nu} A_{\mu}-i\left(A_{\mu} * A_{\nu}-A_{\nu} * A_{\mu}\right) .
$$

We introduce here the spacetime noncommutativity, $\left[\hat{x}^{1}, \hat{x}^{0}\right]=-i \theta$, where we take $\theta>0$ without loss of generality. The equation of motion reads

$$
D_{\mu} F^{\mu \nu}=0
$$

or explicitly

$$
D_{0} F_{01}=D_{1} F_{01}=0
$$

A class of solutions of 2.7) may be obtained by recalling some static solutions of $2+1$ dimensional $\mathrm{U}(1)$ gauge theory on a spatially noncommutative plane $\left[\hat{x}^{1}, \hat{x}^{2}\right]=-i \theta$. The equation of motion satisfied by static configurations is $D_{1} F_{12}=D_{2} F_{12}=0$, which becomes exactly the same as (2.7) by replacing $x^{2} \rightarrow x^{0}$ and $A_{2} \rightarrow A_{0}$. (Note that the equation (2.7) is insensitive to the signature of the metric in two dimensions.) In Ref. 10, 11, solutions carrying magnetic flux are constructed. In the operator form, the solution reads

$$
\hat{A}_{i}=-\frac{1}{\theta} \epsilon_{i j}\left(\hat{x}^{j}-\hat{S} \hat{x}^{j} \hat{S}^{\dagger}\right), \quad(i, j=1,2)
$$

where the shift operator $\hat{S}$ is defined by

$$
\hat{S}^{\dagger} \hat{S}=1, \quad \hat{S} \hat{S}^{\dagger}=1-\hat{P}
$$

with any projection operator $\hat{P}$ satisfying $\hat{P}^{2}=\hat{P}$. The field strength is

$$
\hat{F}_{12}=\frac{1}{\theta} \hat{P}
$$

For simplicity, we take the solution $F_{12}=\frac{1}{\theta}|0\rangle\langle 0|$ with the choice $S=\sum_{n=0}^{\infty}|n+1\rangle\langle n|$ and use it to get the solution of the Lorentzian theory with spacetime noncommutativity. Mapping the solution to function form, one finds that the field strength is localized in spacetime as

$$
F_{10}=\frac{2}{\theta} \exp \left[-\frac{x^{2}+t^{2}}{\theta}\right] .
$$

One may find similar solutions for $1+1$ dimensional Abelian-Higgs theory on the noncommutative spacetime. Adding a fundamental scalar with quartic potential, the action for the Abelian-Higgs theory becomes

$$
S=-\frac{1}{g^{2}} \int d^{2} x\left(\frac{1}{4} F_{\mu \nu} * F^{\mu \nu}+D_{\mu} \phi *\left(D^{\mu} \phi\right)^{\dagger}+\lambda\left(\phi * \phi^{\dagger}-v^{2}\right)^{2}\right)
$$

where $D_{\mu} \phi=\partial_{\mu} \phi-i A_{\mu} * \phi$. The gauge field configuration (2.8) with $\phi=0$ still solves the equations of motion but with Higgs on the local maximum of the potential. One can show that the noncommutative vortex solution found in 11] for spatial noncommutativity solves the above $1+1$ dimensional Abelian-Higgs theory by replacing $x^{2} \rightarrow x^{0}$ and $A_{2} \rightarrow A_{0}$. Note that the vortex solution satisfies $D_{1} F_{12}=D_{2} F_{12}=0, D_{\mu} \phi=0$ and $V^{\prime}(\phi)=0$, 
which makes the equation of motion hold. Again these equations are not sensitive to the signature of the metric.

Solutions localized in spacetime can still be constructed if one adds an adjoint real scalar coupled to the U(1) gauge fields. The action is similar to (2.12) but now with more general potential and the following covariant derivative:

$$
D_{\mu} \phi=\partial_{\mu} \phi-i\left(A_{\mu} * \phi-\phi * A_{\mu}\right) \text {. }
$$

Keeping the gauge field solution as in the previous ones (i.e. with $\hat{F}_{10}=\frac{1}{\theta} \hat{P}$ ), we wish to solve the equations of motion

$$
\begin{aligned}
& D_{\mu} F^{\mu \nu}=-i\left(\phi * D^{\nu} \phi-D^{\nu} \phi * \phi\right), \\
& D_{\mu} D^{\mu} \phi=V^{\prime}(\phi) .
\end{aligned}
$$

To this end, it suffices to find $\phi$ such that $D_{\mu} \phi=0$ and $V^{\prime}(\phi)=0$. Using the solution (2.8), the former equation leads to the following condition,

$$
\left[\hat{S} \hat{x}^{\mu} \hat{S}^{\dagger}, \hat{\phi}\right]=0 \text {. }
$$

This is solved by $\hat{\phi}=\hat{\phi}_{K}$ where $\hat{\phi}_{K} \in \operatorname{Ker} S^{\dagger}$ i.e. $\hat{S}^{\dagger} \hat{\phi}_{K}=0$. In case of nonvanishing potential, we have to find the solution of $V^{\prime}(\phi)=0$ by choosing suitable $\phi_{K}$, which is known for a large class of potentials 18]. If there is no potential, the above ansatz solves the field equations. For instance, $\hat{F}_{10}=\frac{1}{\theta}|0\rangle\langle 0|$ and $\hat{\phi}=a|0\rangle\langle 0|$ corresponds to such a solution. Translating into the function language, it is clear that the solution again has a Gaussian shape localized in spacetime.

So far we have discussed various solutions localized in $1+1$ dimensional spacetime, but such localized solutions may also exist in higher dimensional spacetime noncommutative theories. For example, one may consider $3+1$ dimensional $U(1)$ gauge theory with $\left[x^{\mu}, x^{\nu}\right]=i \theta^{\mu \nu}$. There are substantial differences depending on the signatures of two Lorentz invariants,

$$
\theta^{\mu \nu} \theta_{\mu \nu}=2\left(\left|\vec{\theta}_{m}\right|^{2}-\left|\vec{\theta}_{e}\right|^{2}\right), \quad \epsilon_{\alpha \beta \gamma \delta} \theta^{\alpha \beta} \theta^{\gamma \delta}=8 \vec{\theta}_{e} \cdot \vec{\theta}_{m} .
$$

If $\vec{\theta}_{e} \cdot \vec{\theta}_{m} \neq 0$, one can make a suitable Lorentz transformation to set $\left[\hat{x}^{0}, \hat{x}^{1}\right]=i \theta$, $\left[\hat{x}^{2}, \hat{x}^{3}\right]=-i \theta^{\prime}$ with all the other pairs commuting. One can find localized solutions in $3+1$ dimensional spacetime by a slight generalization of the expression (2.8):

$$
\hat{A}_{\mu}=-\theta_{\mu \nu}^{-1}\left(\hat{x}^{\nu}-\hat{S} \hat{x}^{\nu} \hat{S}^{\dagger}\right), \quad \hat{S}^{\dagger} \hat{S}=1, \quad \hat{S} \hat{S}^{\dagger}=1-\hat{P} .
$$

Again $\hat{P}$ is any projection operator, now acting on a Hilbert space spanned by eigenstates

$$
|m, n\rangle \equiv \frac{\left(c^{\dagger}\right)^{m}}{\sqrt{m !}} \frac{\left(\tilde{c}^{\dagger}\right)^{n}}{\sqrt{n !}}|0,0\rangle \quad\left(\text { where } c=\frac{x_{1}-i x_{0}}{\sqrt{2 \theta}}, \tilde{c}=\frac{x_{2}-i x_{3}}{\sqrt{2 \theta^{\prime}}}\right)
$$

of double harmonic oscillators. The above solution solves the equation of motion (2.6) with either choice of the metric; Lorentzian or Euclidean. The field strength is now given by 
$\hat{F}_{\mu \nu}=\theta_{\mu \nu}^{-1} \hat{P}$. With the simple choice $\hat{P}=|0,0\rangle\langle 0,0|$, we get the field strength localized in spacetime as

$$
F_{10}=\frac{4}{\theta} \exp \left[-\frac{t^{2}+x^{2}}{\theta}-\frac{y^{2}+z^{2}}{\theta^{\prime}}\right], F_{23}=\frac{4}{\theta^{\prime}} \exp \left[-\frac{t^{2}+x^{2}}{\theta}-\frac{y^{2}+z^{2}}{\theta^{\prime}}\right],
$$

while other components are zero.

Coupling a real adjoint scalar to the gauge field, again one may obtain solutions where the matter part is localized in spacetime too. The procedure is very similar to the $1+1$ dimensional case with adjoint scalar, where the scalar satisfies $D_{\mu} \phi=0$.

For the case $\vec{\theta}_{e} \cdot \vec{\theta}_{m}=0$, one has to consider the sign of another invariant. $\vec{\theta}_{e}^{2}<\vec{\theta}_{m}^{2}$ yields the usual spatial noncommutativity (i.e. $\vec{\theta}_{e}=0$ ) by suitable Lorentz transformation, so let us turn to the other two cases.

If $\vec{\theta}_{e}^{2}>\vec{\theta}_{m}^{2}$, one can make a Lorentz transformation to the frame where $\left[x_{0}, x_{1}\right]=i \theta$ while other pairs commute. Simply using the solution (2.11), we get a solution localized in time direction; a 2-dimensional transient 'sheet' occurring at $t=0$.

For the lightlike case $\vec{\theta}_{e}^{2}=\vec{\theta}_{m}^{2}[2,19]$, one can make a Lorentz transformation to a frame where only $\left[x^{-}, x^{2}\right]=i \theta$ is nonzero with $x^{ \pm} \equiv x^{0} \pm x^{1}$. We found a large class of solutions to the Maxwell equation for this case, but none of them is localized in the lightcone time $x^{+}$direction.

In the solutions constructed above, the fields become exponentially small as $|t| \rightarrow \infty$. These may be regarded as spacetime localized degrees arising from the free vacuum. (See also the related argument in the next section.) One may also ask if it is possible to find such a spacetime localized fluctuation that approaches asymptotically a scattering state instead of the vacuum. We shall construct such classical solutions, which will verify explicitly their existence.

First we consider the $1+1$ dimensional $U(1)$ theory. Without matter, there would be no solution that contains scattering states because there is no transverse photon in the $1+1$ dimensions. With a real adjoint scalar, it can be shown that there exist solutions,

$$
\begin{aligned}
& \hat{A}_{\mu}=-\frac{1}{\theta} \epsilon_{\mu \nu}\left(\hat{x}^{\nu}-\hat{S} \hat{x}^{\nu} \hat{S}^{\dagger}\right), \\
& \hat{\phi}=\hat{S} \operatorname{Re}\left(\phi_{0} e^{-i p_{\mu} \hat{x}^{\mu}}\right) \hat{S}^{\dagger}
\end{aligned}
$$

where $p_{\mu} p^{\mu}=0$ and $\phi_{0}$ is any complex number. The first line of the solution $(2.20)$ describes the localized fluctuation whereas the second line contains a plane wave traveling with momentum $p^{\mu}$. One may rigorously show that it approaches asymptotically a plane wave. For instance, choosing $\hat{S}=\sum_{n=0}^{\infty}|n+1\rangle\langle n|$, we have $\hat{P}=|0\rangle\langle 0|$. In functional representation, this is localized near $x^{2}=x_{0}^{2}+x_{1}^{2}<\theta$. Hence, in the asymptotic region $x \gg \sqrt{\theta}$, one expects that $S(x) * S(x)^{*} \approx 1$ up to $\mathcal{O}\left(\frac{\theta}{x^{2}}\right)$ corrections, which implies that $S(x)$ is unitary asymptotically. By an explicit computation, one finds that

$$
\begin{aligned}
& S(x)=e^{-i \tan ^{-1}\left(\frac{x_{0}}{x_{1}}\right)}\left(1+\mathcal{O}\left(\frac{\theta}{x^{2}}\right)\right), \\
& \phi(x)=S(x) * e^{-i p \cdot x} * S(x)^{*}=e^{-i p \cdot x}\left(1+\mathcal{O}\left(\theta \frac{p \cdot x}{x^{2}}\right)\right) .
\end{aligned}
$$


It is clear that $(2.20)$ is a plane wave for sufficiently large $x^{2}\left(=x_{0}^{2}-x_{1}^{2}\right)$. In fact using an appropriate gauge transformation, the power-law corrections may be removed completely, for the initial surface at a large negative time, leaving only exponential corrections.

This kind of solution is not restricted to lower dimensional theories. For the $3+1$ dimensional $U(1)$ theory, let us consider the case $\vec{\theta}_{e} \cdot \vec{\theta}_{m} \neq 0$ first. One can find an exact solution:

$$
\hat{A}_{\mu}=-\theta_{\mu \nu}^{-1}\left(\hat{x}^{\nu}-\hat{S} \hat{x}^{\nu} \hat{S}^{\dagger}\right)+\hat{S} \operatorname{Re}\left(\varepsilon_{\mu} e^{-i p_{\nu} \hat{x}^{\nu}}\right) \hat{S}^{\dagger},
$$

where $p_{\mu} p^{\mu}=\varepsilon_{\mu} p^{\mu}=0$. This solution describes an asymptotic photon with momentum $p_{\mu}$ and polarization $\varepsilon_{\mu}$. As for $\vec{\theta}_{e} \cdot \vec{\theta}_{m}=0$ with $\vec{\theta}_{e}^{2}>\vec{\theta}_{m}^{2}$, we consider the case where only $\left[x_{0}, x_{1}\right]=i \theta$ is noncommutative. It can be shown that there exists a solution

$$
\begin{aligned}
& \hat{A}_{\mu}=-\frac{1}{\theta} \epsilon_{\mu \nu}\left(\hat{x}^{\nu}-\hat{S} \hat{x}^{\nu} \hat{S}^{\dagger}\right)+\hat{S} \operatorname{Re}\left(\varepsilon_{\mu} e^{-i\left(p_{\mu} \hat{x}^{\mu}+p_{k} x^{k}\right)}\right) \hat{S}^{\dagger}, \\
& \hat{A}_{j}=\hat{S} \operatorname{Re}\left(\varepsilon_{j} e^{-i\left(p_{\mu} \hat{x}^{\mu}+p_{k} x^{k}\right)}\right) \hat{S}^{\dagger},
\end{aligned}
$$

with $p^{2}=p_{\mu} p^{\mu}+p_{i} p_{i}=0$ and $p \cdot \varepsilon=p_{\mu} \varepsilon^{\mu}+p_{i} \varepsilon_{i}=0 . \quad(\mu, \nu=0,1$ and $i, j=2,3$.) This describes a plane wave superposed with a spacetime localized sheet centered at $x_{0}=x_{1}=0$.

These examples show that spacetime localized fluctuations may arise not just from the free vacuum but also from scattering states.

Finally let us discuss here about gauge invariant observables for the $1+1$ spacetime noncommutative gauge theories 17]. One may in fact show that all the gauge invariant variables take the form of $\int d^{2} x f\left(X^{\mu}\right)$ where $f$ is an arbitrary function [17] of the covariant coordinate defined by $X^{\mu}=x^{\mu}+\theta^{\mu \nu} A_{\nu}$, which transforms covariantly under the gauge transformation. Then for the configuration of spacetime localized solutions, any observable quantities that are finite indicate that the configuration is localized in space and time. For instance, we define the average position by

$$
q^{\mu} \equiv \frac{\int d^{2} x X^{\mu} F_{01}^{2}}{\int d^{2} x F_{01}^{2}}
$$

and the moments 15

$$
\Delta^{k} \equiv \frac{\int d^{2} x\left(X^{\mu}-q^{\mu}\right)^{k} F_{01}^{2}}{\int d^{2} x F_{01}^{2}}
$$

where we average over the action density. On the spacetime localized solutions, one may show that all the higher moments $(k \geq 2)$ vanish. Thus we conclude the configurations are centered at $q^{\mu}$ and localized indeed.

\section{Discussions}

In this note, we construct Lorentzian solutions of spacetime noncommutative gauge theories, which are exponentially localized in time as well as space. Together with the time translational invariance, we argue that the perturbative $S$ matrix based on the free field degrees is problematic. In the $3+1$ dimensional spacetime noncommutative gauge theories, the problem disappears if $\vec{\theta}_{e} \cdot \vec{\theta}_{m}=0$ and $\vec{\theta}_{e}^{2} \leq \vec{\theta}_{m}^{2}$. Otherwise there exist spacetime localized Lorentzian solutions in $3+1$ dimensional gauge theories. 
The situation here is similar to the case of tachyonic instabilities in quantum mechanical system or field theories. To illustrate the point, consider a dynamical system described by $L=\frac{1}{2} \dot{x}^{2}-V(x)$ with a potential

$$
V(x)=\frac{1}{2}\left(-x^{2}+x^{4}\right)
$$

There is a solution $x(t)= \pm \operatorname{sech}\left(t-t_{0}\right)$ with the velocity $v(t)=\mp \operatorname{sech}\left(t-t_{0}\right) \tanh (t-$ $t_{0}$ ) localized exponentially in time. Existence of such solution does not imply that the dynamical system is problematic. Rather it represents a tachyonic instability at $x=0$. Field theories expanded around tachyonic vacuum have a similar problem. For instance, in free field theories with negative mass squared, the norm of certain states is not preserved and leads to unitarity problems. Some states have a time evolution $e^{-i E t}|E\rangle$ with a purely imaginary energy $E= \pm i \sqrt{\left|m^{2}\right|-\vec{k}^{2}}$ for $\vec{k}^{2}<\left|m^{2}\right|$ and norms cannot be preserved.

Likewise, the existence of spacetime localized solutions for the spacetime noncommutative field theories seems related to similar phenomena. Potential instabilities involved here may be argued in the following way. Using the Seiberg-Witten map 20], the spacetime noncommutative (gauge) field theories may be mapped to the equivalent system but without noncommutativity. There the system has a corresponding background electric field. Instabilities may arise due to this background electric field. In this sense, we suspect that our spacetime localized degrees may be closely connected to the phenomena of tachyon condensation $^{1}$ or $\mathrm{S}$ branes[21]. If this is the case, one may further ask about the true vacuum after tachyon condensation.

Despite attractiveness of such interpretation, however, it should be remembered that we do not know how to make sense of the spacetime noncommutative field theories as quantum mechanics. In summary, the perturbative S-matrix based on free in and out states misses degrees of the spacetime localized solutions. To settle the further issues, we need a nonperturbative quantization of the spacetime noncommutative field theories. Further investigation is necessary in this direction.

In this note, we focus our investigation on the gauge theories, which are normally the ones that can be obtained from string theories in the decoupling limit. But as a separate issue, one could ask the existence of spacetime localized solution for the scalar theories with potential. Consider for example $1+1$ dimensional scalar theory with potential $V(\phi)=-\frac{1}{2} \phi^{2}+\frac{1}{4} \phi^{4}$. This is question about whether there exists GMS (GopakumarMinwalla-Strominger) type solution [18] but one of spatial coordinate is replaced by time. In the large $\theta$ limit, one could ignore the kinetic term as in the GMS case and, for example, $\phi=2 \exp \left[-\frac{t^{2}+x^{2}}{\theta}\right]$ will be solution in the limit. However unlike the case of the GMS soliton, if one tries to perturb the above solution for large but finite $\theta$, one may show that the first order perturbative solution cannot be solved. This happens quite generically for other forms of large $\theta$ solution and other forms of potential. We do not know in this pure scalar case whether there exist spacetime localized solutions or not. Further investigation is required in this direction.

\footnotetext{
${ }^{1}$ See Ref. [3] for the related discussions.
} 


\section{Acknowledgments}

We are grateful to Matthew Kleban, Choonkyu Lee, Lenny Susskind, Takao Suyama, Tsukasa Tada, Hyun-Seok Yang and Ho-Ung Yee for useful discussions and conversations. D.B. is supported in part by KOSEF ABRL R14-2003-012-01002-0 and KOSEF R01-2003000-10319-0. S.K. is supported in part by BK21 project of the Ministry of Education, Korea, and the Korea Research Foundation Grant 2001-015-DP0085.

\section{References}

[1] J. Gomis and T. Mehen, "Space-time noncommutative field theories and unitarity," Nucl. Phys. B 591, 265 (2000) [arXiv:hep-th/0005129].

[2] O. Aharony, J. Gomis and T. Mehen, "On theories with light-like noncommutativity," JHEP 0009, 023 (2000) [arXiv:hep-th/0006236].

[3] L. Alvarez-Gaume, J. L. F. Barbon and R. Zwicky, "Remarks on time-space noncommutative field theories," JHEP 0105, 057 (2001) [arXiv:hep-th/0103069].

[4] D. Bahns, S. Doplicher, K. Fredenhagen and G. Piacitelli, "On the unitarity problem in space/time noncommutative theories," Phys. Lett. B 533, 178 (2002) [arXiv:hep-th/0201222].

[5] C. H. Rim and J. H. Yee, "Unitarity in space-time noncommutative field theories," arXiv:hep-th/0205193.

[6] A. Torrielli, "Cutting rules and perturbative unitarity of noncommutative electric-type field theories from string theory," Phys. Rev. D 67, 086010 (2003) [arXiv:hep-th/0207148].

[7] T. Ohl, R. Ruckl and J. Zeiner, "Unitarity of time-like noncommutative gauge theories: The violation of Ward identities in time-ordered perturbation theory," arXiv:hep-th/0309021.

[8] J. Gomis, K. Kamimura and J. Llosa, "Hamiltonian formalism for space-time non-commutative theories," Phys. Rev. D 63, 045003 (2001) [arXiv:hep-th/0006235].

[9] T. C. Cheng, P. M. Ho and M. C. Yeh, "Perturbative approach to higher derivative and nonlocal theories," Nucl. Phys. B 625, 151 (2002) [arXiv:hep-th/0111160].

[10] A. P. Polychronakos, "Flux tube solutions in noncommutative gauge theories," Phys. Lett. B 495, 407 (2000) [arXiv:hep-th/0007043].

[11] D. Bak, "Exact multi-vortex solutions in noncommutative Abelian-Higgs theory," Phys. Lett. B 495, 251 (2000) [arXiv:hep-th/0008204].

[12] M. Aganagic, R. Gopakumar, S. Minwalla and A. Strominger, "Unstable solitons in noncommutative gauge theory," JHEP 0104, 001 (2001) [arXiv:hep-th/0009142].

[13] J. A. Harvey, P. Kraus and F. Larsen, "Exact noncommutative solitons," JHEP 0012, 024 (2000) [arXiv:hep-th/0010060].

[14] D. J. Gross and N. A. Nekrasov, "Solitons in noncommutative gauge theory," JHEP 0103, 044 (2001) [arXiv:hep-th/0010090].

[15] D. Bak, K. M. Lee and J. H. Park, "Noncommutative vortex solitons," Phys. Rev. D 63, 125010 (2001) [arXiv:hep-th/0011099]. 
[16] D. Bak, S. K. Kim, K. S. Soh and J. H. Yee, "Noncommutative Chern-Simons solitons," Phys. Rev. D 64, 025018 (2001) [arXiv:hep-th/0102137]; D. Bak and K. M. Lee, "Noncommutative supersymmetric tubes," Phys. Lett. B 509, 168 (2001) [arXiv:hep-th/0103148].

[17] D. Bak, K. M. Lee and J. H. Park, "Comments on noncommutative gauge theories," Phys. Lett. B 501, 305 (2001) [arXiv:hep-th/0011244].

[18] R. Gopakumar, S. Minwalla and A. Strominger, "Noncommutative solitons," JHEP 0005, 020 (2000) [arXiv:hep-th/0003160].

[19] R. G. Cai and N. Ohta, "Lorentz transformation and light-like noncommutative SYM," JHEP 0010, 036 (2000) [arXiv:hep-th/0008119].

[20] N. Seiberg and E. Witten, "String theory and noncommutative geometry," JHEP 9909, 032 (1999) [arXiv:hep-th/9908142].

[21] M. Gutperle and A. Strominger, "Spacelike branes," JHEP 0204, 018 (2002) [arXiv:hep-th/0202210]. 